\title{
Aneurisma cerebral y migraña crónica, un reto para el clínico
}

\author{
Miguel Angel Medina-Pabón \\ Médico. Universidad Industrial de Santander (UIS). Colombia \\ Epidemiología. Universidad CES (Corporación para Estudios en la Salud). Medellín. Colombia
}

Recibido: 20/11/2018

Aceptado: 25/02/2019

En línea: 30/04/2019

Citar como: Medina Pabón MA. Aneurisma cerebral y migraña crónica, un reto para el clínico. Rev Esp Casos Clin Med Intern (RECCMI). 2019 (Abr); 4(1): 18-20. doi: 10.32818/reccmi.a4n1a7.

Autor para correspondencia: Miguel Angel Medina-Pabón. michael.medpa@gmail.com

\begin{aligned} Palabras clave \\ \hline$\triangleright$ Migraña crónica \\ $\triangleright$ Aneurisma cerebral \\ $\triangleright$ Cefalea por abuso \\ $\quad$ de analgésicos \\ $\triangleright$ Arteria cerebral anterior \\ $\triangleright$ Cefalea \end{aligned}

\section{Keywords}

$\triangleright$ Chronic migraine

$\triangleright$ Cerebral aneurysm

$\triangleright$ Headache due to analgesic abuse

$\triangleright$ Anterior cerebral artery

$\triangleright$ Headache

\begin{abstract}
Resumen
La ruptura de un aneurisma cerebral es una enfermedad grave que puede conllevar un pronóstico ominoso en caso de no diagnosticarse tempranamente. El aneurisma cerebral en el paciente con migraña crónica y cefalea por abuso de analgésicos es poco frecuente, pero su aparición conlleva una confusión diagnóstica. Presento el caso de una paciente adulta mayor, fumadora, multiconsultante por sus crisis migrañosas crónicas, con cambio de patrón de su cefalea, que requirió neuroimagen y terapia endovascular por una aneurisma sacular en la arteria cerebral anterior derecha sin complicaciones. En conclusión, el rol de la clínica y la neuroimagen son la clave para diagnosticar posible aneurisma cerebral.

Abstract
The rupture of a cerebral aneurysm is a serious illness that can lead to a disturbing prognosis if it is not diagnosed
in time. Cerebral aneurysm in patients with chronic migraine and headache due to analgesic abuse is rare, but
its appearance leads to diagnostic confusion. I present the case of an elderly adult patient, smoker, multi-consul-
tant for chronic migraine attacks, with a change in the pattern of her headache, which required neuroimaging
and endovascular therapy for a saccular aneurysm in the right anterior cerebral artery without complications. In
conclusion, the role of the clinic and neuroimaging are the key to diagnose a possible brain aneurysm.
\end{abstract}

\section{Puntos destacados}

$\triangleright$ Ante signos clínicos de cefalea súbita, cambios de patrón de la cefalea, dolor que despierta en la noche, se debe solicitar neuroimagen para descartar aneurisma cerebral.

$\triangleright$ Como clínicos, hay que insistir en la idoneidad de la historia clínica completa, registrando todo tipo de dolor de cabeza, factores de riesgo, para su diagnóstico certero y tratamiento seguro.

\section{Introducción}

El aneurisma cerebral es una enfermedad poco común, con una prevalencia del 3\% en la población general, de causa desconocida, donde participan elementos bioquímicos y biomecánicos ${ }^{1,2}$. La incidencia de los aneurismas rotos es de alrededor de 9-15 casos por cada 100,000 personas por año en Estados Unidos, especialmente en mujeres, en edades comprendidas entre 30-60 años. En la población latinoamericana, su prevalencia es del $4 \%$ y durante la niñez es del $2 \%$. El $12 \%$ de los pacientes fallece antes de llegar al hospital, el 30-50\% fallece en los primeros 30 días y en la mitad de los supervivientes queda algún tipo de secuela neurológica irreversible $e^{3-5}$.

Los factores de riesgo para la ruptura de un aneurisma son: hipertensión arterial, abuso de alcohol, abuso de cocaína y tabaquismo. Además, el estado y el tamaño del aneurisma afectan al riesgo de ruptura ${ }^{6,7}$. Los síntomas más frecuentes reportados en series de casos son la cefalea intensa de inicio súbito en los que presentaron hemorragia subaracnoidea, y la cefalea migrañosa en menor proporción en los que se había realizado diagnóstico ${ }^{5,8,9}$. A continuación, se informa un caso de aneurisma sacular de la arteria cerebral anterior en un paciente que consultó en múltiples ocasiones por migraña crónica en la Clínica Regional del Valle de Aburra, Policía Nacional (Envigado, Colombia).

\section{Exposición del caso}

Paciente femenina de 68 años de edad, de origen caucásico, ama de casa, con antecedentes de migraña crónica desde los 28 años, con reiteradas consultas por 
cefaleas intensas en Urgencias y consulta externa, la cual es remitida al programa de cefalea de la misma clínica. La cefalea, en los últimos 6 meses, es de tipo picada "calambre o corrientazos" en forma de una diadema que se irradia de la región temporal derecha a la izquierda, la despertaba en la noche, de intensidad moderada 8/10, duración de 1-2 segundos, 4-5 episodios por mes y sin otros síntomas autonómicos ni signos neurológicos.

- Interrogatorio por aparatos. Cefalea migrañosa crónica (hemicránea, tipo pulsátil, asociada a náuseas, mareos, fotofobia y sonofobia, de intensidad moderada a severa, más de ocho episodios por mes, en los últimos 3 meses), cefalea por abuso de analgésicos (debido a que tomaba acetaminofén con codeína, ibuprofeno, tramadol, fencafem; más de 15 dosis de cada uno al mes).

- Hábitos tóxicos. Fumadora de 10-20 cigarrillos al día por 30 años, tomadora ocasional de licor (aguardiente) cada 3-6 meses,

- Antecedentes personales. Hipotiroidismo, osteoporosis, enfermedad pulmonar obstructiva crónica (EPOC) desde hace 10 años.

- Antecedentes obstétricos. Madre de tres hijos, parto vaginal sin abortos (G3P3A0COV3).

- Tomando calcio con vitamina D, alendronato, levotiroxina, calcitriol, inhaladores bucales, inhalador de ipratropol + fenoterol, salbutamol, formoterol.

- Exploración física. En consulta externa, paciente con adecuado estado general, sin signos de focalización, con signos vitales estables, sin soplos a la auscultación de las carótidas ni cardíaco, con resto de exploración sin hallazgos de interés.

- Pruebas diagnósticas. Por presentar signos de bandera roja en los últimos 6 meses (cambio de patrón de la cefalea, la despierta en la noche, edad > 50 años, tabaquismo), se solicitó tomografía axial computarizada (TAC) cerebral simple y contrastada ambulatoria (Figura 1). La TAC cerebral simple fue normal y en la contrastada se evidenció una aneurisma sacular de $6.7 \mathrm{~mm}$ del segmento anterior A3 de la arteria cerebral anterior derecha, sin evidencia de sangrado al momento del estudio.
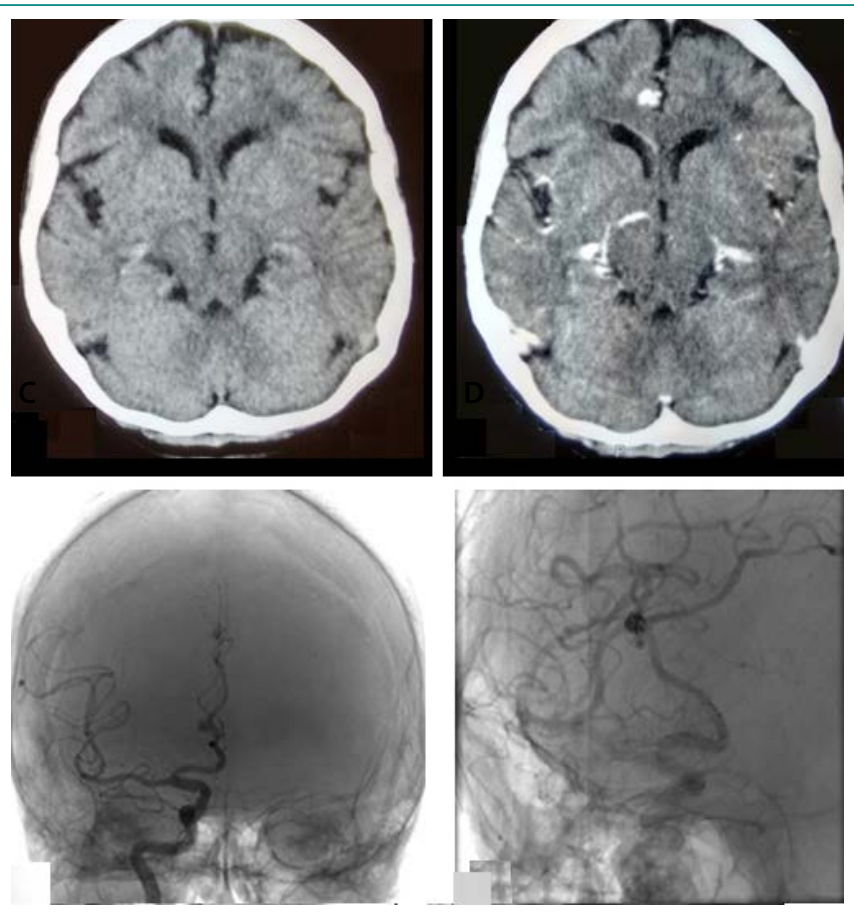

Figura 1. A: TAC simple, dentro de límites normales. B: TAC contrastada, con aneurisma en forma de roseta o sacular en la arteria cerebral anterior derecha, sin presencia de sangrado. C y D: angiografía cerebral; posterior a la terapia endovascular con presencia de coils que ocupan el segmento anterior A3 de la arteria cerebral anterior derecha
Se remitió a Urgencias para manejo por Neurocirugía, y fue intervenida con terapia endovascular sin complicaciones, con mejoría clínica de su cefalea. Actualmente con tratamiento preventivo para la migraña con propranolol 40 mg/12 h, y en controles anuales por Neurocirugía, sin ninguna novedad.

\section{Discusión y conclusiones}

Los aneurismas cerebrales no rotos tienen presentaciones clínicas inespecíicas, en ocasiones se asocian con migraña crónica, prestándose confusiones para el clínico al momento de realizar neuroimagen, asociándose con una elevada morbimortalidad al paciente que sobrelleve el aneurisma y no se le diagnostique. No obstante el diagnóstico diferencial de la cefalea migrañosa puede ser difícil, especialmente de la migraña con aura. En primer lugar, algunas enfermedades pueden producir síntomas similares a la migraña (aneurisma cerebral antes de la ruptura, síndrome de vasoconstricción cerebral reversible). En segundo lugar, algunos trastornos presentan mecanismos fisiopatológicos comunes (p. ej., síndromes CADASIL y MELAS, síndrome antifosfolípido). Y por último, las presentaciones clínicas de migraña a menudo son difíciles de distinguir de las características de otras cefaleas (migraña con aura -ataque isquémico transitorio, migraña con aura visual-, epilepsia occipital) ${ }^{10}$.

Este caso trató de una mujer de la sexta década de la vida, con factores de riesgo vasculares, tabaquismo, alcohol, con cefalea inespecífica, en la cual se realizó estudio de neuroimagen por sospecha de cefalea secundaria. Por tanto, es importante registrar todos los datos en la historia clínica, donde se especifique: tipos de cefaleas, cambios súbitos del patrón de la cefalea frecuente, si presenta cefalea trueno, cefalea que no mejora con analgésicos, cefalea con signos de focalización, cefalea que la despierte en la noche, cefalea súbita con pérdida de consciencia, cefalea precipitada por la actividad física o maniobra de Valsalva, primer inicio del dolor de cabeza $\geq 50$ años de edad, convulsiones, fiebre o traumas severos, los cuales hacen parte de los signos de alarma para solicitar una neuroimagen ${ }^{11,12}$. Posteriormente, se realizó manejo quirúrgico endovascular emergente, obteniéndose mejoría clínica del dolor al colocar profilaxis para la migraña y ausencia completa de la cefalea inespecífica que presentaba.

En conclusión, ante un paciente anciano con cefalea migrañosa que presente factores de riesgo cardiovasculares, que cumpla con signos de bandera roja y un examen físico neurológico normal, se debe solicitar neuroimagen para descartar posibles cefaleas secundarias, como lo es el aneurisma cerebral, buscando siempre el beneficio para el paciente y evitando desenlaces tórpidos o fatales ${ }^{13}$.

\section{Bibliografía}

1. Leclerc JL, García JM, Diller MA, Carpenter AM, Kamat PK, Hoh BL, et al. A comparison of pathophysiology in humans and rodent models of subarachnoid hemorrhage. Front Mol Neurosci [Internet]. 22 de marzo de 2018 [citado 11 de julio de 2018]; 11. Disponible en: http://journal. frontiersin.org/article/10.3389/fnmol.2018.00071/full

2. Sadasivan C, Fiorella DJ, Woo HH, Lieber BB. Physical factors effecting cerebral aneurysm pathophysiology. Ann Biomed Eng. 2013 Jul; 41 (7): $1347-$ 1365. doi: 10.1007/s10439-013-0800-z.

3. Etminan N, Buchholz BA, Dreier R, Bruc P, Torner JC, Steiger HJ, Hänggi D, Macdonald RL. Cerebral aneurysms: formation, progression, and developmental chronology. Transl Stroke Res. 2014 Apr; 5(2): 167-173. doi: 10.1007/ s12975-013-0294-X

4. Lago A, López-Cuevas R, Tembl Jl, Fortea G, Górriz D, Aparici F, Parkhutik $\mathrm{V}$. Tendencies in cerebral aneurism treatment: analysis of a hospital series. Neurología. 2017 Jul-Aug; 32(6): 371-376. doi: 10.1016/j.nrl.2015.12.011. 
5. Zhao M, Liu CS, Xu XY, Xiao YP, Fang C. Unruptured saccular aneurysm presenting migraine. Genet Mol Res. 2014 Jan 24; 13(2): 4046-4049. doi 10.4238/2014.January.24.19.

6. Zhao L, Zhang L, Zhang X, Li Z, Tian L, Wang YX. An analysis of 1256 case of sporadic ruptured cerebral aneurysm in a single Chinese institution. PLoS One. 2014 Jan 15; 9(1): e85668. doi: 10.1371/journal.pone.0085668.

7. Zhou G, Zhu Y, Yin Y, Su M, Li M. Association of wall shear stress with intracranial aneurysm rupture: systematic review and meta-analysis. Sci Rep. 2017 Jul 13; 7(1): 5331. doi: 10.1038/s41598-017-05886-w.

8. Asiltürk M, Abdallah A. Clinical outcomes of multiple aneurysms microsurgical clipping: evaluation of 90 patients. Neurol Neurochir Pol. 2018 Jan-Feb; 52(1): 15-24. doi: 10.1016/j.pjnns.2017.09.005
9. Lebedeva ER, Busygina AV, Kolotvinov VS, Sakovich VP, Olesen J. Remission of migraine after clipping of saccular intracranial aneurysms. Acta Neurol Scand. 2015 Feb; 131(2): 120-126. doi: 10.1111/ane.12292.

10. Sergeev AV. Migraine 'masks': differential diagnosis of acute headache. Zh Nevrol Psikhiatr Im S S Korsakova. 2018; 118(1): 96-102. doi: 10.17116/ jnevro20181181196-102.

11. Mitsikostas DD, Ashina M, Craven A, Diener HC, Goadsby PJ, Ferrari MD, et al. J Headache Pain. 2015; 17: 5. doi: 10.1186/s10194-016-0596-y.

12. Mier RW, Dhadwal S. Primary headaches. Dent Clin North Am. 2018 Oct; 62(4): 611-628. doi: 10.1016/j.cden.2018.06.006

13. Starling AJ. Diagnosis and management of headache in older adults. Mayo Clin Proc. 2018 Feb; 93(2): 252-262. doi: 10.1016/j.mayocp.2017.12.002. 